\title{
Desenvolvimento da Vigilância Epidemiológica de Fronteira no contexto da Globalização: conceitos e marcos teóricos.
}

\section{Development of Border Epidemiological Surveillance in the context of globalization: concepts and benchmarks.}

\section{Desarrollo de la Vigilancia Epidemiológica de la Frontera en el contexto de la globalización: conceptos y marcos teóricos.}

\author{
Robson BRUNIERA-OLIVEIRA ${ }^{1}$ \\ Marco Aurelio Pereira HORTA ${ }^{2}$ \\ Vinícius Silva BELO ${ }^{2}$ \\ Eduardo Hage CARMO ${ }^{3}$ \\ José Fernando de Souza VERANI ${ }^{4}$
}

RESUMO: Objetivo. Apresentar e analisar as ações desenvolvidas para a implementação e desenvolvimento de Vigilância Epidemiológica de Fronteiras, nos Blocos Econômicos Multinacionais, particularmente na América do Sul e Brasil. Metodologia. Foi realizado um estudo de revisão narrativa incluindo artigos científicos, documentos técnicos, diretrizes, normas, manuais, pautas de reuniões ou qualquer outro documento que fosse relacionado ao desenvolvimento e implementação de ações e programas de Vigilância Epidemiológica em Fronteiras. O estudo foi baseado nos sites das instituições/organizações multinacionais e nas bases MedLine, PubMed, Scielo e Scopus. Resultados. Foram identificados a criação de órgãos e ações no intuito de implementar e desenvolver a vigilância epidemiológica de fronteiras para os seguintes blocos: União Europeia (Health Security Committee, European Center for Disease Prevention and Control, Sistema de alerta rápido e resposta, General Rapid Alert System e Rede de Monitoramento Ad Hoc); Tratado NorteAmericano de Livre Comércio (Border Infectious Disease Surveillance, "Projeto de Vigilância

1 Médico Veterinário formado na Universidade Federal de Viçosa, Mestre em Epidemiologia em Saúde Publica pela Escola Nacional de Saúde Publica Sergio Arouca ENSP/FIOCRUZ. Possui ênfase nas áreas de Epidemiologia, Vigilância Epidemiológica, Saúde Publica e Zoonoses. Contato: Robson.Bruniera@gmail.com

2 Escola Nacional de Saúde Pública - ENSP Fundação Oswaldo Cruz - FIOCRUZ, Rio de Janeiro. Brasil

3 Instituto Suramericano de Gobierno en Salud - ISAGS/UNASUR

4 Escola Nacional de Saúde Pública - ENSP Fundação Oswaldo Cruz - FIOCRUZ, Rio de Janeiro. Brasil 
e Alerta Rápido de Doenças Infeciosas") ; Mercado Comum do Sul ( Reunião de Ministros da Saúde do Mercosul, Subgrupo de Trabalho 11 Saúde e Subcomissão Controle Sanitário de Portos, Aeroportos, Terminais e Passos Fronteiriços Terrestres); e União de Nações Sul-Americanas. Conclusões. Os programas e politicas desenvolvidos até o momento refletem o reconhecimento da importância do assunto por parte dos Estados Membros da OMS. Uma maior integração, com troca de informações, incluindo fluxos, frameworks, planos de preparação e o fortalecimento de áreas estratégicas como de energia e transporte deve ser incentivada intra e inter blocos. Palavras chaves: Vigilância Epidemiológica. Controle Sanitário de Fronteiras. Doenças Transmissíveis

ABSTRACT: Objective. To analyze the actions for implementation and development of Epidemiological Surveillance of Borders in Multinationals trade bloc, particularly in South America and Brazil, to suggestnew actions to aiming to improve current programs. Methods. Anarrative review of documents was conducted including scientific articles, technical documents, guidelines, manuals, agendas of meetings or any other document that was related to development and implementation of epidemiological surveillance at Borders. The study was based on the websites of the institutions / multinationals organizations and in the MedLine, PubMed, Scopus and SciELO. Results. It was identified the establishment of agencies and actions in order to implement and to develop border surveillance for the following trade blocs: European Union (Health Security Committee, European Centre for Disease Prevention and Control, Rapid Alert System and response, General Rapid Alert System and a Monitoring Ad Hoc Network), the North American Free Trade Agreement (Border Infectious Disease Surveillance, "Project Monitoring and Early Warning Infectious Disease"), the Southern Common Market (the Meeting of Ministers of Health of MERCOSUR (RMS), 11 Health Sub-Working Group, Subcommittee and Sanitary Control of Ports, Airports, Terminals and Steps Frontier Land) and Union South American Nations. Conclusion. The programs and policies developed reflect the recognition of importance of the subject by the WHO Member States, since the world is moving forward to higher connectivity and integration. Greater integration with exchange of information, including flows, frameworks, preparation plans and strengthening of strategic areas such as energy and transportation should be promoted within and between blocs. Key Words: Epidemiological Surveillance. Sanitary Control of Borders. Communicable Diseases.

RESUMEN: Objetivo. Presentar y analizar las medidas adoptadas para la aplicación y desarrollo de la vigilancia epidemiológica de las Fronteras, en Multinacionales Bloques Económicos, particularmente en América del Sur y Brasil. Método. Fue realizado un estudio de la revisión narrativa de literatura, incluyendo artículos científicos, documentos técnicos, directrices, normas, manuales, agendas de reuniones o cualquier otro documento que esté relacionado con el desarrollo y la implementación de acciones y programas de Vigilancia Epidemiológica de las fronteras. El estudio se basó en las páginas web de las instituciones / organizaciones y multinacionales, y en las bases de datos Medline, PubMed, Scopus y SciELO. Resultados. Fue identificada la creación de órganos y acciones con el fin de poner en práctica y desarrollar la vigilancia epidemiológica 
de fronteras para los siguientes bloques: Unión Europea (Health Security Committee, European Centre for Disease Prevention and Control, Sistema de alerta rápida y de las respuestas, General Rapid Alert System y la Red de Monitoreo Ad Hoc); El Tratado de Libre Comercio de América del Norte (Border Infectious Disease Surveillance, "Projeto de Vigilância e Alerta Rápido de Doenças Infeciosas"); El Mercado Común del Sur (Reunión de Ministros de Salud del MERCOSUR, el Subgrupo de Trabajo No 11 "Salud" y la Subcomisión de Puertos, Aeropuertos, Terminales y Puntos de Frontera); y la Unión de Naciones Suramericanas. Conclusión. Los programas y las políticas desarrolladas hasta la fecha, reflejan el reconocimiento de la importancia de la concesión por los Estados Miembros de la OMS. Una mayor integración con el intercambio de información, incluidas las series, los marcos, los planes de preparación y el fortalecimiento de áreas estratégicas como de la energía y el transporte deben ser incentivados intra e inter bloques.

Palabras clave: Vigilancia Epidemiológica. Control Sanitario de Fronteras. Enfermedades Transmisibles.

\section{INTRODUÇÃO}

Desde o final do século vinte vivemos um momento ímpar na organização mundial, na qual os países estão reduzindo suas barreiras físicas e taxas alfandegárias, criando assim blocos econômicos multinacionais, com zonas de livre comércio ${ }^{(1)}$. Com a abertura dos mercados, o volume de comercializações de produtos aumentou consideravelmente, além do aumento e garantia da livre circulação de pessoas e serviços, intra e entre os blocos. Isso, associado ao desenvolvimento de tecnologias de comunicações e transporte, resultou em uma constante mobilização de grandes volumes de pessoas e bens entre países e continentes em um período muito curto ${ }^{(1)}$. Com a diminuição das barreiras espaciais e temporais, tornou-se possível viajar entre diversas partes do mundo em um tempo inferior ao período de incubação de muitas doenças infecciosas. Em tal contexto, as fronteiras dos países tornaram-se abertas para a circulação e entrada de doenças infecciosas emergentes e reemergentes, assim como seus vetores ${ }^{(2)}$.

Fronteiras, no contexto da vigilância epidemiológica, podem ser definidas como marcos que dividem espaços físicos, criando assim diferentes unidades geopolíticas. Essas divisões, no entanto, não são absolutas e nem são capazes de impedir as interações sociais, os fenômenos naturais, as práticas religiosas, os movimentos culturais e a propagação de doenças e epidemias ${ }^{(3)}$. Espaços fronteiriços são locais de características especificas, com grandes heterogeneidades geográficas, de aspectos históricos, culturais, étnicos, econômicos e sociais. Além disso, caracterizam-se frequentemente por intensos fluxos populacionais, o que gera condições particulares para a transmissão de doenças ${ }^{(4)}$.

O intenso movimento de pessoas, animais e bens são componentes importantes na distribuição de doenças infecciosas no mundo. O movimento de pessoas facilitou a transmissão de doenças contagiosas, como o vírus HIV e o vírus da Influenza H1N1, que tiveram sua disseminação amplificada principalmente pelo transporte aéreo. Doenças que possuem vetores em seu ciclo podem 
ser introduzidas, assim como o agente quanto o vetor, em novas áreas geográficas por viajantes, navios e aviões ${ }^{(2)}$. A introdução da Febre Amarela nas Américas por meio de embarcações de escravos foi um exemplo: os navios negreiros traziam escravos com o vírus e grandes quantidades do vetor, o Aedes aegypti ${ }^{(5)}$. Do mesmo modo, o movimento de animais, sejam de companhia, produção, selvagens ou de produtos derivados, tem sua relevância. Animais têm sido identificados como sendo a origem de muitas das recentes doenças emergentes identificadas, incluindo os vírus da HIV/AIDS, H5N1 Influenza Aviaria, H1N1 influenza A e a Síndrome Respiratória Aguda Grave (SARS) ${ }^{(2)}$. Das 335 novas doenças infecciosas identificadas no mundo nos últimos anos, 60.3\% são zoonoses. Dessas, mais de $70 \%$, tiveram sua origem em animais selvagens ${ }^{(6)}$.

Assim, a Vigilância Global de Saúde ganha atualmente caráter crucial no monitoramento de emergências relacionadas à Saúde Pública Internacional ${ }^{(7)}$. Para fazer face a essa nova situação global, no ano de 2007, entrou em vigor o novo Regulamento Sanitário Internacional (RSI), que introduziu a notificação à Organização Mundial da Saúde (OMS) de todo e qualquer evento que constitua uma Emergência de Saúde Pública Internacional (ESPI), assim como a implementação de qualquer recomendação daquela organização para lidar com a emergência ${ }^{(7)}$. Outro ponto relevante do RSI revisado é o estabelecimento da obrigatoriedade, entre os Estados-Membros, do desenvolvimento de sua capacidade nacional e regional de vigilância epidemiológica, ou seja, da capacidade de detectar e responder a ameaças à saúde pública, com ênfase na disseminação dos dados nacional e internacionalmente em tempo real ${ }^{(7)}$.

Em razão da especificidade das áreas fronteiriças e por se caracterizarem como regiões estratégicas para o controle de agravos, o desenvolvimento de politicas e ações direcionadas para essas regiões ganham caráter crucial nos programas de saúde de qualquer país ou bloco. Esse artigo tem por objetivo apresentar e discutir conceitos como o de bloco econômicos multinacionais, e as ações de VE de fronteiras desenvolvidas por cada bloco, como marcos teórico da vigilância epidemiológica de fronteiras, particularmente no MERCOSUL e Brasil, com intuito de fazer uma analise dos programas já desenvolvidos, ressaltando os pontos positivos de cada programa, lições depreendidas e sugerir possíveis novas ações para melhorar os atuais programas em curso no Brasil e América do Sul.

\section{MÉTODO}

Foi realizado um estudo de revisão narrativa incluindo artigos científicos, documentos técnicos, diretrizes, normas, manuais, pautas de reuniões ou quaisquer outros documentos que fossem relacionados ao desenvolvimento e implementação de ações de Vigilância Epidemiológica em Fronteiras. $\mathrm{O}$ estudo foi baseado nos sites das instituições/organizações multinacionais e nas bases MedLine, PubMed, Scielo e Scopus, restringindo a pesquisa às publicações nas línguas inglesa, espanhola e portuguesa, entre os anos de 1990 e 2013. Adotou-se para essa revisão o conceito de blocos econômicos multinacionais, em que são identificadas as ações, politicas, mecanismos e órgãos comuns a todos os países pertencentes a cada um destes: União Europeia, Tratado Norte- 
Americano de Livre Comércio, Mercado Comum do Sul e União de Nações Sul-Americanas (Unasul) - os quais apresentam até o momento, politicas mais sólidas já implementadas. Realizouse uma análise particularizada e qualitativa dos sistemas de vigilância encontradas, sendo os resultados discriminados por blocos econômicos. Existem outros tipos vigilâncias Epidemiológicas multinacionais entre diferentes países, não vinculadas a blocos econômicos, por isso não foram alvo desse estudo.

\section{RESULTADOS}

\section{Principais Blocos criados e Ações Desenvolvidas}

\section{União Europeia}

A criação do Mercado Comum Europeu, em 1992, eliminou barreiras alfandegárias entre os Estados-Membros, configurando a União Europeia (UE) com uma população de quase 500 milhões de pessoas, tornando-se o maior bloco econômico do mundo ${ }^{(8)}$. Em razão da proximidade dos países, do intenso fluxo de pessoas e bens, e dos diferentes status sanitários entre eles, a UE é uma área vulnerável para a entrada de diversas ameaças transfronteiriças de diversas naturezas, com potencial para causar severas consequências para a população ${ }^{(9)}$. Embora a gestão das crises de saúde pública em nível nacional seja da competência de cada Estado-Membro, nenhum país pode enfrentar sozinho uma crise de saúde pública transfronteiriça. A base jurídica para a abordagem das ameaças sanitárias transfronteiriças graves foi reforçada com o Tratado de Lisboa ${ }^{(10) .}$ Esse Tratado, determinou que a UE deve complementar e apoiar as políticas nacionais e incentivar a cooperação entre os Estados-Membros ${ }^{(10)}$, de modo a garantir a uniformidade das ações e evitar que outros Estados-Membros sejam prejudicados diante ações adotadas por um ou mais países. Por meio de uma comissão (Comissão Europeia) foram criadas mecanismos, instrumentos e políticas para coordenar as ações dos Estados-membros no enfrentamento das ameaças à segurança da saúde de uma forma eficaz e coerente. Assim, quando um Estado-Membro pretende adotar medidas de saúde para combater uma ameaça, esse deve, antes de adoptar tais ações, consultar os outros Estados-Membros e a Comissão sobre a natureza, finalidade e alcance das medidas, a menos que a necessidade de proteger a saúde pública seja tão urgente que a adoção imediata das medidas se faça necessária ${ }^{(11-13)}$.

Dentro das ações, políticas, mecanismos e instrumentos para atender a prevenção e controle de graves ameaças transfronteiriças desenvolvidas pela União, podemos destacar: Health Security Committee (HSC), European Centre for Disease Prevention and Control (ECDC), Sistema de Alerta Rápido e de Resposta (SARR), General Rapid Alert System (ARGUS) e uma Rede de Monitoramento Ad Hoc (Tabela 1).

O Health Security Committee (HSC) foi criado pelos ministros de saúde dos países da UE após os ataques terroristas de Setembro de 2001, quando foi utilizada a toxina Antraz. Inicialmente o 
mandato do HSC era limitado ao bioterrorismo, tendo logo se expandido para outras questões de saúde ${ }^{(11)}$. O Comitê fornece uma plataforma para a troca informal de informações e experiências sobre estratégias de preparação e gestão de crises, aconselhando ministros da Saúde e a Comissão Europeia no planejamento e resposta, além de coordenar o planejamento de emergência. Em seu escopo, três áreas são enfocadas: prevenção, preparação e respostas às ameaças à saúde. Seu foco de atuação é principalmente no plano de preparação e resposta, viabilizando a interoperabilidade de preparação dos planos nacionais; a implementação consistente das necessidades de capacidade do núcleo de vigilância e resposta;e a garantia do fornecimento das informações a respeito de preparação e resposta à Comissão pelo Estados-Membros ${ }^{(13)}$.

\begin{tabular}{|c|c|}
\hline Órgão & Objetivo/Ação \\
\hline Health Security Committee (HSC) & - Gerenciar ameaças graves transfronteiças \\
\hline $\begin{array}{c}\text { European Center for Disease } \\
\text { Prevention and Control (ECDC) }\end{array}$ & $\begin{array}{l}\text { - Realizar a vigilância, detecção e avaliação dos riscos } \\
\text { de ameaças para a saúde humana decorrentes de doenças } \\
\text { transmissíveis e de surtos de origem desconhecida }\end{array}$ \\
\hline $\begin{array}{c}\text { General Rapid Alert System } \\
\text { (ARGUS) }\end{array}$ & $\begin{array}{l}\text { Fornecer uma plataforma interna para o intercambio, } \\
\text { das informações sobre crises emergentes multissetoriais ou } \\
\text { ameaça previsível ou iminente; } \\
\text { - Disponibilizar um processo de coordenação } \\
\text { adequado para ser ativado em caso de grandes crises; } \\
\text { - Fornecer o contexto para comunicar-se com os } \\
\text { cidadãos e oferecer uma imagem equilibrada, coerente e } \\
\text { completa dos esforços desenvolvidos pela Comissão }\end{array}$ \\
\hline Rede de Monitoramento Ad Hoc & $\begin{array}{l}\text { - Monitorar e detectar a ocorrência de alterações } \\
\text { nos padrões de distribuição geográfica, transmissão e } \\
\text { severidade na ocorrência dos agravos por meio dos sistemas } \\
\text { de informações }\end{array}$ \\
\hline
\end{tabular}

Em abril de 2004, foi criado o European Centre for Disease Prevention and Control (ECDC), sendo conferido a esse um mandato que abrange a vigilância, detecção e avaliação dos riscos de ameaças para a saúde humana decorrentes de doenças transmissíveis e de surtos de origem desconhecida. Tal centro tem se encarregando de conduzir a vigilância epidemiológica das doenças transmissíveis e de coordenar o funcionamento do Sistema de Alerta Rápido e de Resposta, além de dar suporte cientifico e técnico a Comissão.

O General Rapid Alert System (ARGUS) foi criado pela Comissão Europeia com o objetivo de fornecer uma plataforma interna para o intercambio, em tempo real, das informações relevantes sobre as crises emergentes multissetoriais ou ameaça previsível ou iminente; tornar disponível um processo de coordenação adequado para ser ativado em caso de grandes crises; e fornecer o contexto para comunicar, de forma eficaz, com os cidadãos e oferecer uma imagem equilibrada, coerente 
e completa dos esforços desenvolvidos pela Comissão. O ARGUS complementa os outros setores do Sistema de Alerta Rápido que opera em caso de crises multissetoriais. Este sistema mantem comunicação permanente entre a Comissão e as autoridades competentes em nível nacional para alertar, avaliar os riscos de saúde pública e determinar quais medidas podem ser necessárias para proteger a saúde pública ${ }^{(13)}$.

A rede de Monitoramento Ad hoc é uma cooperação entre os Estados-Membros no intuito de monitorar e detectar a ocorrência de alterações nos padrões de distribuição geográfica, transmissão e severidade na ocorrência dos agravos por meio dos sistemas de informações. A rede entra em operação apenas em situações nas quais é reconhecida uma ameaça transfronteiça grave para a saúde ou com potencial de rápida dispersão entre os Estados-Membros. Essa rede, estabelecida pela Comissão Europeia, faz a comunicação entre a Comissão e os pontos focais de cada EstadoMembro, monitorando os dados coletados no intuito de processar e gerar informações e subsidiar ações. De modo a garantir a comparação e compatibilidade dos dados coletados, dentro da União, e a adoção de processos genéricos para a operação da rede, a comissão por sua vez, faz, se necessário, as atualizações das definições de casos ${ }^{(13)}$.

\section{Tratado Norte-Americano de Livre Comércio}

O Tratado Norte-Americano de Livre Comércio ou NAFTA é um tratado que envolve Canadá, México e Estados Unidos da América e tem o Chile como associado, em uma atmosfera de livre comércio, com custo reduzido para troca de mercadorias entre os três países. Ele entrou em vigor em $1^{\circ}$ de janeiro de 1994 e possui um prazo de 15 anos para a total eliminação das barreiras alfandegárias entre os três países da América do Norte. Seus principais objetivos são: eliminar as barreiras alfandegárias, e facilitar o movimento de produtos e serviços entre os territórios dos países participantes; promover condições para uma competição justa dentro da área de livre comércio; aumentar substancialmente as oportunidades de investimento dos países participantes; oferecer proteção efetiva e adequada e garantir os direitos de propriedade intelectual no território de cada um dos países participantes; criar procedimentos efetivos para a implementação e aplicação deste tratado, para sua administração conjunta e para a resolução de disputas e estabelecer uma estrutura para futura cooperação trilateral, regional e multilateral para expandir e realçar os benefícios deste acordo ${ }^{(14)}$.

Os Estados Unidos e México têm uma das mais importantes relações binacionais do mundo. Os dois países compartilham uma fronteira de $3.2000 \mathrm{~km}$ e estão profundamente ligados economicamente e culturalmente. A região da fronteira tem uma população estimada em aproximadamente 11 milhões de pessoas, muitos dos quais a atravessam diariamente ${ }^{(15)}$. Em vista dessa proximidade e do volume de pessoas e bens que atravessam a fronteira, foram desenvolvidas ações com o intuito de prevenir e reduzir o impacto de uma ESPI na saúde (Tabela 2). No ano de 1997, a Associação de Saúde da Fronteira dos Estados Unidos-México e o Conselho dos EUA de Epidemiologistas do Estado e Território aprovaram resoluções para apoiar a vigilância de doenças 
infecciosas emergentes ao longo da fronteira EUA-México. O Centers for Disease Control and Prevention (CDC), em conjunto com a Secretaria da Saúde do México, estabeleceu o Border Infectious Disease Surveillance (BIDS), criando assim uma cooperação na área de epidemiologia (15). O BIDS é o primeiro sistema de vigilância sindrômica de doenças infecciosas binacional, com o objetivo de formar parcerias entre as instituições municipais, estaduais e federais e autoridades de saúde pública da região de fronteira, para aumentar a operacionalidade e oportunidade das ações de vigilância epidemiológica, diminuindo a lacuna na vigilância de doenças na fronteira, como a incompatibilidade de definições de casos utilizados nos dois países e o aumento da capacidade de diagnostico laboratorial da região.

Outro programa criado para região de fronteira foi o "Projeto de Vigilância e Alerta Rápido de Doenças Infeciosas (PVARDI)". Financiado pelo Departamento de Saúde e Serviços Humanos do EUA e envolvendo 20 dos 21 estados que fazem fronteira com EUA-México e EUA-Canada, esse foi iniciado no ano de 2004 visando melhorar as atividades transfronteiriças na detecção precoce, identificação e notificação de doenças infecciosas associadas com potencial de agentes de bioterrorismo ou que possam ser consideradas riscos para a Saúde Pública. O projeto representa uma colaboração única em nível estadual, federal e internacional, que desenvolve suas atividades de forma a melhorar a rápida e efetiva confirmação laboratorial de casos urgentes reportados de doenças infecciosas nas regiões fronteiriças dos Estados Unidos, Canadá e México. Dentro de seu escopo, as atividades do PVARDI focam a melhoraria da coordenação entre os Estados vizinhos entre os EUA e o México; a melhora as capacidades de vigilância no local, estadual e internacional; o inicio oportuno de investigações epidemiológicas; o compartilhamento de dados, incluindo dados laboratoriais; e a adequada capacitação dos profissionais de saúde da região ${ }^{(16)}$.

\begin{tabular}{|c|l|}
\hline \multicolumn{2}{|c|}{ Tabela 2 - Principais órgãos, } \\
\hline Órgãos & \multicolumn{1}{c|}{ Objetivo/Ação } \\
\hline $\begin{array}{c}\text { Border Infectious Disease } \\
\text { Surveillance }\end{array}$ & $\begin{array}{l}\text { Formar parcerias entre as instituições municipais, } \\
\text { estaduais e federais e autoridades de saúde pública na } \\
\text { região de fronteira, para aumentar a operacionalidade e } \\
\text { oportunidade das ações de vigilância epidemiológica }\end{array}$ \\
\hline $\begin{array}{c}\text { Projeto de Vigilância e Alerta } \\
\text { Rápido de Doenças Infeciosas } \\
\text { (PVARDI) }\end{array}$ & $\begin{array}{l}\text { Melhorar as atividades transfronteiriças na } \\
\text { detecção precoce, identificação e notificação de doenças } \\
\text { infecciosas associadas com potencial de agentes de } \\
\text { bioterrorismo ou que possam ser consideradas ameaças } \\
\text { para a Saúde Pública }\end{array}$ \\
\hline
\end{tabular}

\section{Mercado Comum do Sul}

O Mercado Comum do Sul ou MERCOSUL é a união aduaneira de cinco países da América do Sul, criado no ano de 1991 com a assinatura do Tratado de Assunção. Inicialmente composto por 4 países: Argentina, Brasil, Paraguai e Uruguai ${ }^{(17)}$. Em 2006, a Venezuela deu início ao processo 
de adesão ao bloco. Esse acordo permitiu a livre circulação de bens, serviços e fatores de produção entre os países integrantes, além da possibilidade de se buscar por acordos com diferentes blocos como a UE. O MERCOSUL se tornou o quarto maior mercado consumidor do mundo, com um PIB que ultrapassa 3 trilhões de dólares ${ }^{(17,18)}$. O processo de integração de fronteiras do Cone Sul latinoamericano trouxe consigo uma intensificação do fluxo de pessoas e comércio. Assim, a região de fronteira passou a ser entendida como ponto crítico desse processo, e uma área estratégica para a integração sul-americana. Por isso, políticas nacionais que priorizam seu desenvolvimento vêm ganhando maior espaço ${ }^{(1)}$.

$\mathrm{Na}$ área de saúde, o Mercosul está organizado no entorno de dois foros, sendo eles a Reunião de Ministros da Saúde do Mercosul (RMS) e o Subgrupo de Trabalho 11 Saúde (SGT 11 Saúde). A RMS tem por objetivo propor medidas para a coordenação de políticas de saúde no Mercosul. É uma instância de cooperação técnica regional para formular planos, programas, estratégias e diretrizes. Ela está organizada em comissões intergovernamentais que são integradas por dirigentes, especialistas e técnicos representantes dos países, que discutem temas de interesse nacionais. No ano de 2005 foi criada a comissão intergovernamental que é responsável por discutir a implementação, revisão e implantação do regulamento sanitário internacional ${ }^{(19)}$.

O Subgrupo de Trabalho 11 Saúde possui o objetivo de "harmonizar as legislações dos Estados Partes referentes aos bens, serviços, matérias-primas e produtos da área da saúde, os critérios para a vigilância epidemiológica e controle sanitário com a finalidade de promover e proteger a saúde e a vida das pessoas e eliminar os obstáculos ao comércio regional, contribuindo dessa maneira ao processo de integração" ${ }^{(19)}$. Ele é composto por três Comissões: Vigilância em Saúde, Produtos para a Saúde e Serviços de Atenção à Saúde. Essas comissões são organizadas e, subcomissões e grupos ad hoc orientados por programas de trabalhos anuais. Dentro da comissão de vigilância em saúde, localiza-se a Subcomissão Controle Sanitário de Portos, Aeroportos, Terminais e Passos Fronteiriços Terrestres, onde as ações e politicas para essas áreas são discutidas. Essa subcomissão visa trabalhar no tema de fronteiras para que os países envolvidos desenvolvam e implementem de forma adequada o RSI, com o desenvolvimento de suas capacidades de vigilância e elaboração de planos e guias para a resposta as ESPIN e ESPI; uniformização das definições de agravos; e a facilitação da troca de informação em saúde, dinamizando o processo de resposta entre as partes envolvidas $^{(20-22)}$.

\section{União de Nações Sul-Americanas (Unasul)}

A União de Nações Sul-Americanas (Unasul) é uma iniciativa político-estratégica de relacionamento sul-americano que visa aprimorar o diálogo diplomático e as formas mais variadas de intercâmbio e colaboração nos campos político, econômico, social e cultural. Composta por 12 países-membros (Argentina, Bolívia, Brasil, Chile, Colômbia, Equador, Guiana, Paraguai, Peru, Suriname, Uruguai e Venezuela), conta ainda com dois observadores (México e Panamá), a União possui como objetivo "construir, de forma participativa e consensual, um espaço de integração e 
união nos campos cultural, social, econômico e político entre seus povos, outorgando prioridade ao diálogo político, às políticas sociais, saúde, educação, energia, infraestrutura, financiamento e meio ambiente, entre outros, com vistas a eliminar as desigualdades socioeconômicas, lograr a inclusão social e a participação cidadã, fortalecer a democracia e reduzir as assimetrias, no marco do fortalecimento da soberania e independência dos Estados membros" (23).

Criada em 2009 foi pelos Chefes de Estado dos países membros, a Unsaul entrou em plena vigência a partir de março de 2011, quando o Uruguai tornou-se o nono país a ratificá-lo e depositálo na Secretaria Geral. Atualmente o bloco possui status de observador na Assembleia Geral das Nações Unidas ${ }^{(24)}$. Essa união intergovernamental possibilitou a integração de duas uniões alfandegárias preexistentes: MERCOSUL e Comunidade Andina. Desse modo, a diplomacia regional tem por primícias identificar as oportunidades e negociar os acordos nas áreas estruturantes que representem maior prioridade, incluindo as áreas da Saúde ${ }^{(25)}$.

A Unasul esta estruturada com os seguintes componentes: Conselho dos Chefes de Estados e Governo, representando a instância mais elevada; Conselho de Ministros das Relações Exteriores, que formulam as propostas submetidas à decisão do Conselho e toma as decisões executivas; Conselho dos Delegados composto por funcionários governamentais de alto nível dedicados a organizar o trabalho dos dois Conselhos superiores e a implementar suas decisões; e Conselhos Sul-Americanos da Defesa, Desenvolvimento Social, Educação, Cultura, Ciência, Tecnologia e Inovação e Saúde, este ultimo também conhecido como Conselho de Saúde Sul-Americana (Unasul Saúde). A presidência do bloco é temporária, denominada Presidência Pro Tempore (PPP), sendo regida por um ano e alternada entre os países-membros ${ }^{(25)}$.

O Conselho de Saúde Sul-Americana (Unasul Saúde) é um órgão permanente composto pelos ministros da Saúde dos países membros, com o objetivo de "Consolidar a América do Sul como um espaço de integração em saúde contribuindo para a Saúde de todos e desenvolvimento, incorporação e integração dos esforços e conquistas do MERCOSUL, ORAS CONHU e ACT". Destaca-se entre os objetivos específicos a priorização das ações do Unasul Saúde nas áreas de fronteira ${ }^{(26)}$.

Suas ações são orientadas pelo Plano Estratégico Quinquenal 2010-2015, criado levando em conta a situação de saúde da América do Sul e esta organizado em torno de cinco eixos ${ }^{(27)}$. 1. Política Sul-americana de Vigilância e Controle de Eventos em Saúde; 2. Sistemas universais de saúde; 3. Acesso universal a medicamentos e outros insumos para a saúde e desenvolvimento do complexo produtivo da saúde na América do Sul; 4. Promoção da saúde e ações sobre os determinantes sociais da saúde; 5 . Desenvolvimento de recursos humanos em saúde. No âmbito de uma iniciativa regional como a Unasul-Saúde, será enfatizado o cuidado especial com a saúde nas fronteiras, incluindo a garantia de portabilidade para o acesso aos serviços de saúde de qualquer dos países-membros de sul-americanos não residentes no país em que procuram assistência. A Presidência do Conselho é exercida pelo Ministro ou Ministro da Saúde do mesmo país que exerce 
a presidência Pro Tempore da Unasul ${ }^{(25)}$.

Entre as principais ações desenvolvidas pela Unasul-Saúde, podemos destacar na área de integração de vigilância de fronteiras o Instituto Sul-Americano de Governo em Saúde (ISAGS). Este foi criado no ano de 2010 e se caracteriza como uma entidade intergovernamental de caráter público, integrante do Conselho de Saúde Sul-Americano da União das Nações Sul-Americanas, que tem como principal objetivo promover o intercâmbio, reflexão crítica, gestão do conhecimento e a geração de inovações no campo da política e governança em saúde entre os países da América do Sul. Suas principais funções são a gestão e produção do conhecimento; desenvolvimento de lideranças; e assessoramento técnico colocando à disposição dos Ministérios da Saúde da América do Sul as melhores práticas e evidências que qualificam a gestão em saúde. Essa entidade dedicase também ao desenvolvimento de lideranças em sistemas, serviços, organizações e programas na área da saúde, assim como ao apoio técnico às instituições de governo ${ }^{(28)}$.

\section{Brasil}

O Brasil, país membro do bloco econômico MERCOSUL, possui características que o tornam um país vulnerável à entrada de Emergências em Saúde Publica (ESP), o que pode provocar sérias consequências para saúde pública e a economia ${ }^{(29,30)}$. Ele possui uma grande extensão de fronteiras terrestres com nove países com diferentes status sanitários, além de diversos portos e aeroportos, com intensa movimentação comercial e fluxo de pessoas. Sua fronteira (franja fronteiriça) possui $15,719 \mathrm{~km}$ de extensão e ocupando uma área de $2.300 .000 \mathrm{~km}^{2}(27,6 \%$ do território nacional), com 588 municípios e quase 10 milhões de habitantes (6\% da população nacional) ${ }^{(31)}$. Essa área é marcada por uma grande diversidade social, cultural e econômica, e por isso foi dividida em três distintas macrorregiões, arco norte, central e sul. O arco norte é o maior com $1.524 .156 \mathrm{~km}^{2}(66,7 \%$ da superfície da franja) e o menos povoado, ocupando apenas $15,7 \%$ da franja, sendo que $37,8 \%$ vivem em zonas rurais. As populações indígenas nessa área são significativas, e excedem a população não indígena em alguns os municípios. Esse arco apresenta as piores condições de vida de toda a fronteira, possuindo os piores indicadores de expectativa de vida e mortalidade infantil. Isso pode ser atribuído a falta de infraestrutura, recursos humanos e baixa cobertura da saúde na região ${ }^{(31)}$.

$\mathrm{O}$ arco central da fronteira apresenta duas situações distintas. Na parte norte do arco, as condições são semelhantes ao arco do Norte, enquanto que ao sul, a situação é mais perto daqueles encontrados no arco sul. $\mathrm{O}$ arco sul é a região mais populosa da fronteira, com 403 municípios, apresenta um elevado número de pequenas propriedades rurais, o que resulta em uma população mais estável. Possui as melhores condições de vida, sendo que a expectativa de vida supera aos 70 anos e a mortalidade infantil é inferior a 30 óbitos por mil nascidos vivos, refletindo uma maior disponibilidade de serviços de saúde ${ }^{(31)}$.

Em virtude dessa vulnerabilidade, politicas e programas na área da vigilância epidemiológica de 
$86 / /$

fronteira devem ser estimuladas e desenvolvidas no país. De acordo com a avaliação do sistema de vigilância brasileiro, conduzido no ano de 2009, com foco em identificar as capacidades essenciais, deficiências e limitações em lidar com emergências de saúde pública, foi identificado que em todos os três níveis de governo, o sistema de vigilância brasileiro possui planos legais bem estabelecidos (incluindo regulamentos técnicos essenciais), infraestrutura, insumos, materiais e mecanismos necessários para coordenação. No entanto, ainda há alguns pontos fracos em nível estadual, especialmente em áreas de fronteira e em cidades de pequeno porte ${ }^{(32)}$.

Embora algumas atividades e políticas voltadas para as fronteiras tenham sido identificadas, até o momento no Brasil, não foi criado nenhum sistema de vigilância epidemiológica de forma sólida que análise a população da fronteira como uma unidade geográfica ${ }^{(15)}$. Algumas inciativas foram propostas e implementadas pelos governos de modo aumentar a capacidade de vigilância e reposta as ESPIN: Centro de Informações Estratégicas em Vigilância em Saúde (CIEVS), Força Nacional do Sistema Único de Saúde e a Rede de Laboratórios de Fronteira (Tabela 3).

O Centro de Informações Estratégicas em Vigilância em Saúde (Cievs), em funcionamento desde o ano de 2006, possui a função de detectar emergências de saúde pública, propiciar o gerenciamento dessas emergências e coordenar resposta apropriada pelo Ministério da Saúde, junto com as secretarias estaduais e municipais de saúde. Operando continuamente, o centro amplia a capacidade de detecção e resposta a qualquer emergência de saúde pública de importância nacional, caracterizando-se como uma ferramenta fundamental para o enfrentamento de pandemias, fortalecendo a capacidade de resposta já existente ${ }^{(33)}$. Outra ação desenvolvida foi a criação da Força Nacional do Sistema Único de Saúde (FN-SUS), visando executar medidas de prevenção e assistência em situações como desastres ou de desassistência às populações em território nacional e internacional.

\begin{tabular}{|c|l|}
\hline \multicolumn{2}{|c|}{ Tabela 3 - Principais órgãos, mecanismos e ações implementadas pelo MERCOSUL e } \\
Brasil.
\end{tabular}

A Rede de Laboratórios de Fronteiras, coordenada pela Secretaria de Vigilância em Saúde 
(SVS) e composta por 12 laboratórios, foi criada para desenvolver a capacidade e oportunidade de detecção das principais doenças transmissíveis, executar análises, efetuar coletas, armazenar e enviar amostras aos laboratórios de referência ${ }^{(34)}$.

\section{Outras Vigilâncias Epidemiológicas Multinacionais}

A título de ilustração citamos outras vigilâncias multinacionais que desenvolvem suas atividades na área de controle e prevenção de agravos com potencial de espalhassem para as partes envolvidas, sendo essas vigilâncias: CPS - International Circumpolar Surveillance Program; COMISCA Consejo de Ministros de Salud de Centro America; EAIDSN - East African Integrated Disease Surveillance Network; ES - Epi South; MBDSN - Mekong Basin Disease Surveillance; MECIDS Middle East Consortium for ID Surveillance; PPHSN - Pacific PH Surveillance Network; SEEHN - Southeastern European Health Network.

\section{DISCUSSÃO}

É evidente que nenhum país pode enfrentar sozinho quaisquer ameaças transfronteiriças, uma vez que essas têm importantes implicações transnacionais e requerem adequadas planificação e preparação, com avaliação e procedimentos de gestão de riscos. Para assegurar que todos os países estejam igualmente preparados para gerir os impactos em uma crise de saúde pública, ações coordenadas fazem-se necessárias. Dessa maneira, o estabelecimento de uma boa cooperação multinacional e multisetorial são de extrema importância, com planos de preparação e/ou avaliações de risco coerentes entre todos os países que de alguma forma estão envolvidos. A falta de coordenação transnacional pode resultar na adoção de medidas desproporcionais ou atraso na capacidade de resposta, podendo trazer sérias consequências para a saúde e o comércio ${ }^{(10)}$. Portanto, a criação de politicas de saúdes internacionais intra e inter blocos reflete o reconhecimento da vulnerabilidade dos países para entrada de ameaças em seus territórios.

No entanto, apesar das politicas criadas e implementadas pelos blocos para a prevenção e controle das ameaças transfronteiriças, existem ainda limitações a serem vencidas. As politicas implementas foram direcionadas para emergências envolvendo doenças infecciosas. Em virtude desse direcionamento de politicas, nenhum bloco dispõe de planos estruturados para lidar com riscos radio-nucleares, o que caracteriza uma vulnerabilidade em um setor importante para as saúde e economia ${ }^{(10)}$.

A UE, em razão do caráter informal do Comitê de Segurança em Saúde, o qual a participação é voluntária, apresenta um fragilidade no envolvimento e compromisso dos Estados-Membros com as ações desenvolvidas ${ }^{(9)}$. Como resultado, acordos entre os membros são difíceis de serem alcançados e, quando são, os Estados-Membros não são obrigados a levá-los em consideração em sua resposta de saúde pública em situações de crise. Em função da ausência de uma coordenação de preparação da UE, não há uma ligação adequada entre o Regulamento Sanitário Internacional 
(RSI) e a maneira na qual as ameaças graves transfronteiriças para a saúde são abordados pela UE. Isso representa um risco para ao gerenciamento da saúde pública em nível da UE, dado que a implementação de capacidades mínimas essenciais para a vigilância e resposta às emergências de saúde pública de preocupação internacional pelos Estados-Membros se dá de forma não uniforme e inconsistente ${ }^{(9)}$.

Na questão do direcionamento de ações coordenadas em nível da UE, ainda há diversas lacunas como: inconsistente preparação e planejamento de resposta entre Estados Membros da UE para todos os tipos de ameaças graves de saúde transfronteiriças; insuficientes mecanismos de monitoramento e avalição de riscos químicos, biológicos e ameaças ambientais; e fracos procedimentos de comunicação de riscos. Além disso, dentro do plano de planejamento e preparação as emergências, as discrepâncias nos níveis de planejamento da preparação entre os Estados-Membros leva a incoerentes estratégias, normas divergentes e procedimentos e metodologias inconsistentes. Os países membros, atualmente, dispõem de diferentes níveis de capacidades para responder aos riscos, o que pode enfraquecer e atrasar a capacidade de resposta da UE e impactar negativamente a situação em outros noutros Estados-Membros ${ }^{(9)}$.

O Brasil, junto ao MERCOSUL e ao Unasul-Saúde, embora tenham iniciado o desenvolvimento de algumas ações e politicas, ainda possui muitas lacunas a serem preenchidas. A iniciativa dos países membros do MERCOSUL em criar um órgão, no qual problemas e questões de interesses comuns relacionados à saúde possam ser trabalhadas em conjunto, representa um importante passo. O MERCOSUL e a Unasul são modelos de integração diferentes, com origens distintas; o MERCOSUL teve suas origens primordialmente comerciais e no decorrer do desenvolvimento de suas atividades passou a incluir temas sociais em suas pautas. Já a Unasul é um processo diz respeito ao diálogo entre os países da América do Sul. Com isso, os grupos responsáveis pelas saúde e suas decisões, nos dois blocos, diferem consideravelmente ${ }^{(25)}$.

Um importante avanço para os países envolvidos nos programas da América do Sul foi a elaboração e implementação da resolução com a lista de doenças prioritárias para serem notificadas em as partes envolvidas, com suas definições de casos, diagnóstico de laboratório, medidas de controle e prevenção e sistema de informação (registro, consolidação e análise) ${ }^{(35)}$. A falta de definições de agravos entre diferentes países poderia acarretar em retardo na identificação, notificação e desencadeamento de ações para controle. Sérias consequências econômicas e sociais podem advir dessa diminuição de oportunidade de vigilância, em virtude da possibilidade de acometimento de varias nações, com elevado numero de pessoas infectadas e a imposição de importantes embargos econômicos.

No entanto, apesar da criação de uma coordenação multinacional, problemas como as diferenças dos sistemas de saúde e a discrepância entre as capacidades de resposta dos países, principalmente laboratorial, são obstáculos a serem vencidos ${ }^{(34)}$. Para a maioria das doenças infeciosas de importância nacional e internacional a confirmação laboratorial é fundamental para o desencadeamento de ações 
de controle e prevenção. Por isso, a estruturação de uma rede laboratorial que seja capaz de atender a demanda oriunda de estados fronteiriços aumenta a sensibilidade, oportunidade e especificidade do sistema de vigilância em detectar novos agravos e a ocorrência de padrões anormais de eventos e o desencadeamento de ações de respostas ${ }^{(34)}$.

Para dinamizar essa estruturação, a adoção do desenvolvimento de Vigilâncias Epidemiológicas do tipo sindrômicas pode ser considerada uma boa alternativa. Esse modelo de vigilância é definido como uma vigilância que se embasa na coleta sistemática e contínua de dados relacionados à saúde, análise e interpretação de indicadores em tempo real, com o intuito de detecção de surtos de doenças e a garantia uma resposta rápida a Saúde Pública ${ }^{(35)}$. Por informar sobre a existência de surtos, clusters ou mesmo aumento das incidências de doenças de caráter sazonal, a Vigilância Sindrômica (VS) possui potencial de suplementar os sistemas de vigilâncias convencionais ${ }^{(36)}$, sendo considerada uma importante ferramenta no desenvolvimento de capacidades nacionais e regionais de vigilância epidemiológica. A VS ganhou maior importância depois de episódios de atentados de bioterrorismo como os ocorridos nos Estados Unidos da América em que foram criados diversos tipos de VS como forma de aumentar a sensibilidade e oportunidade de detecção de ocorrências de eventos anormais. Em razão desse modelo de vigilância, dentro das áreas delimitadas de fronteiras, as vigilâncias locais dos países desenvolveriam uma logística operacional sindrômica diante de casos suspeitos de ESPI e ESPIN, no qual varias hipóteses diagnosticas são verificadas simultaneamente, aumentando com isso a oportunidade do diagnóstico e do desencadeamento das medidas de bloqueio e prevenção.

Uma questão que sempre deve ser considerada quando se planeja a implementação de qualquer sistema em saúde pública é referente ao custo para implementação e operacionalização dessa vigilância. A dimensão recursos financeiros, pode ser considerada, em todas as fases de um sistema, como sua maior limitação. Entretanto, estudos demostraram apesar dos custos operacionais decorrente de uma vigilância sindrômica em tempo real quando comparado com a não detecção precoce de surtos, os benefícios de uma VS supera o custo financeiros de sua operacionalização ${ }^{(37)}$.

Diante do exposto, a maior integração, com troca de informações, incluindo fluxos, frameworks, planos de preparação e o fortalecimento de áreas estratégicas como de energia e transporte deve ser incentivada intra e inter blocos. Nesse sentido, a perspectiva de trabalho multinacional é essencial para uma efetiva prevenção e controle da transmissão de doenças infecciosas que se movem com facilidade através das fronteiras geopolíticas. Os programas e politicas desenvolvidos até o momento refletem o reconhecimento da importância do assunto por parte dos Estados Membros da OMS, uma vez que o mundo caminha de encontro a maior conexão e integração.

\section{REFERENCIAS BIBLIOGRÁFICAS}

1- Gadelha CAG, Costa L. Integração de fronteiras: a saúde no contexto de uma política nacional de desenvolvimento. Cad. Saúde Pública, Rio de Janeiro 2007, 23 (2):214-226. 
2- Institute of Medicine. Infectious disease movement in a borderless world. Washington, DC: The National Academies Press. 2010.

3- Rodrigues-Júnior, AL. Epidemias na faixa de fronteira brasileira. VII Encontro Nacional de Estudos Estratégicos, Brasília-DF, 2007.

4- Peiter P, Machado O, Iñiguez RL. Doenças transmissíveis na Faixa de Fronteira Amazônica: o caso da malária.In: Miranda AC, BarcellosC, MoreiraJC,MonkenM. Território, ambiente e saúde. Rio de Janeiro, Editora Fiocruz, 2008,p.257-272.

5- Ministério da Saúde. Fundação Nacional de Saúde. Manual de vigilância epidemiológica da febre amarela. Brasília, 1999.

6- Castillo-Salgado C. Trends and Directions of Global Public Health Surveillance. Epidemiol Rev. 2010, 32(1):93-109.

7- Hardiman M, Wilder-Smith A. The revised international health regulations and their relevance to travel medicine. J Travel Med14(3) : 141-144, 2007.

8- União Europeia. [Internet].[acesso em 28/06/2010]. Disponível em: http://europa.eu/ index_pt.htm.

9- European Commission. Commission Staff Working Paper Impact Assessment. Accompanying the document Decision of the European Parliament and of the Council on serious cross-border threats to health. 2011. ].[acesso em 29/06/2010]Disponível em: http://europa.eu/ index_pt.htm.

10- Comissão Europeia. Proposta de decisão do parlamento europeu e do conselho relativa a ameaças sanitárias transfronteiriças graves, 2011. ].[acesso em 29/06/2010] Disponível em: http:// europa.eu/index_pt.htm.

11- Comissão Europeia - Comunicado de Imprensa Saúde Pública: A Comissão propõe medidas eficazes para proteger melhor os cidadãos contra uma vasta gama de ameaças sanitárias transfronteiriças, 2010. ].[acesso em 28/06/2010] Disponível em: http://ec.europa.eu/health/ preparedness_response/policy/index_pt.htm

12- European Commission. Commission Staff Working Document. Health Security in the European Union and Internationally, 2009. ].[acesso em 30/06/2010] Disponível em: http://europa. eu/index_pt.htm.

13- European Commission. Proposal for a decision of the european parliament and of the council on serious cross-border threats to health. 2011. Disponível em: http://europa.eu/index_pt.htm. 
14- North American Free Trade Agreement. NAFTA [Internet], [acesso em 28/06/2010]. Disponível em : http://www.nafta-sec-alena.org

15- Weinberg M, Waterman S, Lucas CA, et al. The U.S.--Mexico Border Infectious Disease Surveillance project: establishing bi-national border surveillance. Emerg Infect Dis 2003;9:97-102.

16- Centers for Disease Control and Prevention. DGMQ Stories: Building Cross-Border Public Health Partnerships, 2011. [acesso em 28/06/2012].Disponível em: www.cdc.gov/migrationhealth

17- Machado MH, Paula AE, Aguiar FW.O trabalho em saúde no MERCOSUL: uma abordagem brasileira sobre a questão. Cad. Saúde Pública, Rio de Janeiro 2007, 23(2):S292-S301.

18- Banco Mundial, World Economic Outlook Database, Outubro de 2007, para o ano de 2006. [Internet], [acesso em 28/06/2013]. Disponível em: http://www.imf.org

19- MERCOSUL/GMC/RES No 06/05 Pauta Negociadora do SGT n 11 "Saúde" (Revogação da RES. GMC No21/01).[Internet], [acesso em 05/09/2013]. Disponível em: http://www.mercosur. int/msweb/portal\%20intermediario/Normas/normas_web/Resoluciones/PT/RES_006-005_PT_ Pauta\%20Neg.PDF.

20- MERCOSUR/SGT N 11/ACTA N 01/13. XL Reunión ordinaria del subgrupo de trbajo n 11 “SALUD”.[Internet],[acesso em 15/10/2013]. Disponível em: http://www.mercosur.int/innovaportal/file/5154/1/sgt11_2013_acta01_es.pdf

21- MERCOSUL. Pauta negociadora do SGT N 11 «Saúde» (revogação da Resolução GMC No 06/05). Resolução No 13/07 do Grupo do Mercado Comum. [Internet], [acesso em 28/06/2010].

22- MERCOSUL/XXVIII SGT No 11/P.RES. N $N^{0}$ 01/07. Pauta negociadora do SGT $\mathrm{N}^{\mathrm{o}} 11$ "Saúde" (revogação da RES. GMC No 06/05). [Internet], [acesso em 05/09/2013]. Disponível em: http://200.214.130.44/mercosulsaude/portugues/sgt/rosgt11/ROSGTXXVIII/ACTA01-07/ ANEXO-IVA/Pauta-PT.pdf

23- União das Nações Sul-Americanas (Unasul). Tratado Constitutivo da União de Nações Sul-Americanas. [Internet], [acesso em 08/08/2013]. Disponível em: http://www.itamaraty.gov. br/sala-de-imprensa/notas- aimprensa/2008/05/23/tratado-constitutivo-da-uniao- de-nacoessul/?searchterm=constituição-unasul

24- União de Nações Sul-Americanas (Unasul). [Internet], [acesso em 08/08/2013]. Disponível em: http://www.isags-nasursalud.org/Default.asp?lang=1

25- Buss, P.M. Cooperacao e integracao regional em saude na America do Sul: A contribuicao da Unasul-Saude Source: Ciência e saúde coletiva 2011, 6(6):2699 -2711. 
26- União de Nações Sul-Americanas (Unasul). Decisión para el Establecimiento del Consejo de Salud Suramericano de la UNASUR. [Internet], [acesso em 10/09/2013]. Disponível em: http:// www.unasursg.org/uploads/ce/cd/cecdfa7cf8540bc4e855e773d1242403/Estatutos-Consejo-deSalud-Suramericano.pdf

27- União de Nações Sul-Americanas (Unasul). Saúde. Plano Quinquenal 2010-2015. [Internet], [acesso em 6/09/2013].Disponível em:http://www.unasursalud.org/images/stories/documentos/ plan_quinquenal_ingles.pdf

28- Brasil. Decreto $n^{\circ} 7.667$, de 11 de janeiro de 2012. Promulga o Tratado Constitutivo da União de Nações Sul-Americanas, firmado em Brasília, em 23 de maio de 2008. acesso em 6/09/2013].Disponível em: http://www.planalto.gov.br/ccivil_03/_Ato2011-2014/2012/Decreto/ D7667.htm

29- Machado LVN, Amin MM. Impacto da febre aftosa na posição competitiva do Brasil no mercado internacional de carne bovina. Sociedade Brasileira de Economia e Sociologia Rural. XLIV CONGRESSO DA SOBER, Fortaleza-CE, 2006.

30- Rodrigues AF, Escobar AL, Souza-Santos R. Análise espacial e determinação de áreas para o controle da malária no Estado de Rondônia. Rev Soc Brasil Med Trop 2008 , 41:55-64.

31- Peiter P.C. Condiciones de vida, situación de la salud y disponibilidad de servicios de salud en La frontera de Brasil: un enfoque geográfico. Cad. Saúde Pública, Rio de Janeiro 2007, 23 (2):237-250.

32- Ministério da Saúde do Brasil. Secretaria Executiva. Sistema Integrado de Saúde das Fronteiras SIS-Fronteira. Relatório de Desempenho do Projeto. Dezembro/2008.

33- Heffernan R. Syndromic surveillance in public health practice, New York City. Emerg Infect Dis 10:858 (2004)

34- Brasil. Portaria n 2.761 , DE 18 de Novembro de 2008. Vigilância epidemiológica e controle de enfermidades priorizadas e surtos entre os estados partes do mercosul. . [Internet], [acesso em 10/08/2013].Disponível em: http://dt2001.saude.gov.br/sas/PORTARIAS/Port2008/GM/GM2761.htm

35- Henning KJ. What is syndromic surveillance? MMWR Morb Mortal Wkly Rep 2004;53(Suppl):5-11.

36- Begier EM, Sockwell D, Branch LM, et al. The National Capitol Region's emergency department syndromic surveillance system: do chief complaint and discharge diagnosis yield different results? Emerg Infect Dis 2003;9:393--6. 
37- Smith RD, Keogh-Brown MR, Barnett T, Tait J. The economy-wide impact of pandemic influenza on the UK: a computable general equilibrium modelling experiment. BMJ. 2009 19;339.

Artigo apresentado em 03/05/14 Artigo aprovado em 03/09/14 Artigo publicado no sistema em 10/10/14 